\title{
Mineralización de Mucuna pruriens y Crotalaria juncea en dos órdenes de suelos en Costa Rica
}

\author{
Albán Arias Quesada ${ }^{1}$, Paola Brenes Rojas² ${ }^{2}$ Laura Sánchez ${ }^{1} \&$ Wagner Peña ${ }^{3}$ \\ 1. TFG Licenciatura Ingeniería Agronómica, Universidad Estatal Distancia; albanarias@gmail.com, \\ lauraelenasv@gmail.com \\ 2. Vicerrectoría de Investigación, Universidad Estatal Distancia; paolarjs@gmail.com \\ 3. Catedra de gestión sostenible del suelo. Escuela de Ciencias Exactas y Naturales. UNED. Universidad Estatal a \\ Distancia. Apartado Postal 474-2050. San Pedro, San José. Costa Rica; wpenaenator@gmail.com
}

Recibido: 22 julio 2017

\section{RESUMEN}

En la mineralización de rastrojos inciden múltiples factores que se pueden ver asociados a los órdenes de suelos. Se establecieron ensayos experimentales en dos sitios diferentes con Ultisol (Valle del General, Pérez Zeledón) y Andisol (San Isidro, Heredia) para analizar la mineralización de Mucuna pruriens y de Crotalaria juncea, donde es afectada por el clima, las características del suelo y el déficit hídrico. Fue observado que el rastrojo de $M$. pruriens aportó aproximadamente $274,13 \mathrm{Kg}$ N/ha en el suelo ultisol y $367 \mathrm{Kg}$ N/ha en el suelo andisol, pero $C$. juncea aportó 159,46 Kg $\mathrm{N} /$ ha en el ultisol y $188,27 \mathrm{Kg}$ N/ha en el suelo andisol; durante la mineralización de cada rastrojo, aumentó el contenido de nitrógeno, en el Ultisol fue de 0,11 a $0,51 \%$ y en el Andisol de 0,21 a 0,56\%., lo que demuestra mejoramiento de la fertilidad del suelo en ambos órdenes de suelos, en especial con rastrojo de $M$. pruriens.

Palabras clave: nitrógeno, leguminosa, suelo.

\begin{abstract}
ABSTRAC
It is to be assumed that the mineralization of legume stubble is different in each order of soil. Experimental trials were established at two different sites with ultisol (Valle del General, Pérez Zeledón) and andisol (San Isidro, Heredia) to analyze the mineralization of Mucuna pruriens and Crotalaria juncea, where it is affected by climate, soil characteristics and The water deficit. It was observed that M. pruriens stubble contributed approximately $274,13 \mathrm{Kg}$ $\mathrm{N} /$ ha in the ultisol soil and $367 \mathrm{KgN} / \mathrm{ha}$ in the andisol soil, but $\mathrm{C}$. juncea contributed $159,46 \mathrm{Kg} \mathrm{N} /$ ha in the ultisol and $188,27 \mathrm{~kg} \mathrm{~N} / \mathrm{ha}$ in soil andisol; During the mineralization of each stubble, the nitrogen content increased, in the ultisol it was of 0.11 to $0.51 \%$ and in the andisol of 0.21 to $0.56 \%$, which demonstrates improvement of soil fertility In both orders of soils, especially with stubble of M. pruriens.
\end{abstract}

Key words: nitrogen, legume, soil.
Aceptado: 26 octubre 2017

\section{Introducción}

En Costa Rica es poco conocido el efecto que tienen los abonos verdes sobre las propiedades químicas, físicas y biológicas del suelo, por lo que una alternativa para minimizar el uso de fertilizantes químicos es la aplicación de abonos verdes que provienen de las plantas de la familia de las leguminosas, ya que por medio de estos abonos se puede mantener o incrementar los niveles de materia orgánica, aportar nutrientes e incrementar la presencia de microorganismos benéficos (Durango, 2014, 137 p).

Debido a las malas prácticas agrícolas empleadas en los suelos dedicados a la producción de cultivos, se produce un constante desgaste de todas sus propiedades afectándose con ello su grado de fertilidad, lo que se traduce en menos rendimientos productivos por cosechas (Prager, M., Sanclemente, O., Sánchez de Prager, M., Miller J. y Ángel, D. 2012, 53-62 p), por lo que se recomienda el uso de abonos orgánicos de leguminosas. Este tema es de especial preocupación para los productores a nivel mundial, ya que debido al constante abuso de este recurso sin las prácticas regenerativas adecuadas, ha generado una disminución de las producciones y una degradación del estado de los suelos.

Aprovechándose del proceso de la mineralización de la aplicación de abonos verdes se puede minimizar el uso de fertilizantes sintéticos usados en los suelos dedicados a la producción agrícola, ya que a través de este proceso biológico que sufren los rastrojos, pueden hacer disponible los nutrimentos que necesitan las plantas para su desarrollo. Este proceso es considerado como una fuente importante de nitrógeno, carbono y demás elementos, pues se transforman las formas orgánicas del elemento a formas 
minerales asimilables, así como también recuperar las características físicas, químicas y bilógicas de los suelos dentro de una práctica de conservación natural (Moreno, 2009, 73 p), contribuyendo así al uso correcto del nutriente en las variables de manejo de los cultivos agrícolas y con ello ayuda a la recuperación de la fertilidad natural de los suelos (Ruiz, 2003, 451 p).

Es por ello que se pretende analizar la mineralización del sustrato de Mucuna pruriens, (Mucuna) y Crotalaria juncea (Crotalaria) como abonos verdes en un suelo Andisol de Heredia y un Ultisol de Pérez Zeledón.

\section{Metodología}

Se caracterizaron dos fincas dedicadas a la producción de café, en San Isidro de Heredia y el Distrito de San Rafael de Platanares en Pérez Zeledón. El diseño experimental fue factorial compuesto de cuatro tratamientos y tres repeticiones, para evaluar la disponibilidad del nitrógeno de las leguminosas Mucuna pruriens y Crotalaria juncea a través de la descomposición y mineralización del rastrojo o sustrato orgánico. Hubo un total de 18 mallas (bolsas) de nylon de 12 x $12 \mathrm{~cm}$ por cada especie, las cuales contenían $50 \mathrm{~g}$ y fueron depositadas a una profundidad de $10 \mathrm{~cm}$ en suelos Andisol y Ultisol, en dos parcelas de 180 $\mathrm{m}^{2}$, terrenos de la finca del señor Hernán Cordero, que se ubica en el cantón de San Isidro de Heredia y la otra parcela que se ubica en el cantón de San Isidro del General, Distrito San Rafael de Platanares en la finca del señor Tobías Gonzáles Jiménez.
Fue aplicado un análisis de Varianza (ANOVA), con el método de comparaciones múltiples LSD Fisher y un nivel de significancia del $0,5 \%$ con el Programa INFOSTAT, VERSION: 2016.

\section{Resultados y Discusión}

En la figura 1, se observó que los tratamientos de la leguminosa Mucuna (T1) incorporados al suelo Andisol, durante los primeros 10 días de haberse establecido el experimento, logró aportar un $0,6 \%$ de nitrógeno aproximadamente con base a su contenido inicial, pero que a partir del día 10 ese contenido de nitrógeno se incrementó de un 3,4\% a 4,6\% aproximadamente, es decir, aumentó el contenido de nitrógeno que proviene de los rastrojos. Es de suponer que el aumento en el contenido de nitrógeno en las muestras durante el proceso de mineralización, se deba al incremento de la superficie específica, debido a su vez por la naturaleza de las arcillas alófanas, que favorecería la teoría de la retención de nutrimentos por una fuerza electroestática.

En cuanto a la mineralización que sufrió los tratamientos la especie Crotalaria (T2), durante los primeros 20 días de establecido el experimento figura 2, la mineralización presentó un proceso lento, ya que hubo muy poca variación entre el porcentaje inicial y los 20 días, pero que después del día 20 hasta el día 30 del experimento, el contenido paso de un $3,2 \%$ a un $2,7 \%$, coincidiendo con (Rincón, 2016 $151 \mathrm{p})$, en donde indica que aquellos rastrojos que presentan una relación $\mathrm{C}: \mathrm{N}$ alta, son más lentos en su proceso de mineralización (Crotalaria: $\mathrm{C}: \mathrm{N}$ de un

\section{Mucuna}

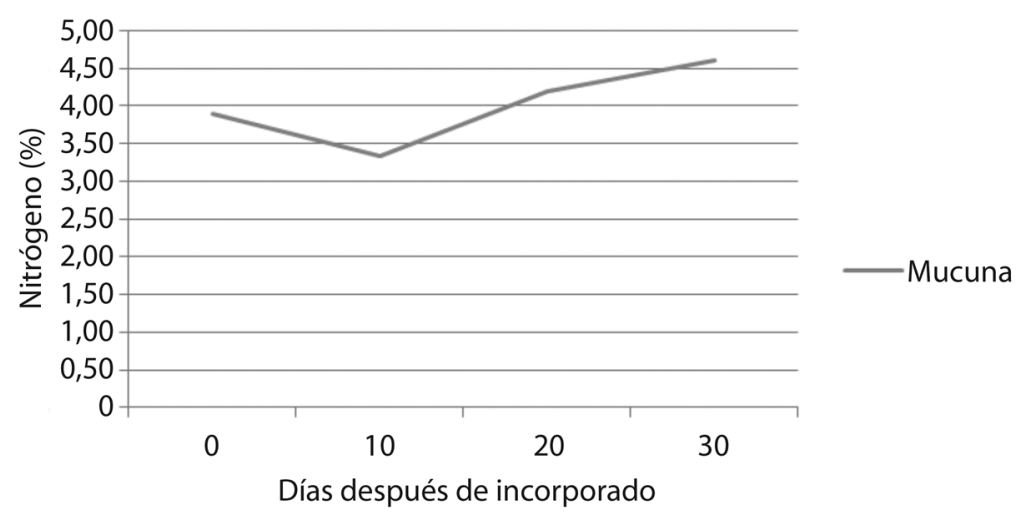

Figura 1. Mineralización de nitrógeno del rastrojo de Mucuna incorporado en un suelo Andisol en Heredia. T1. Mucuna. 


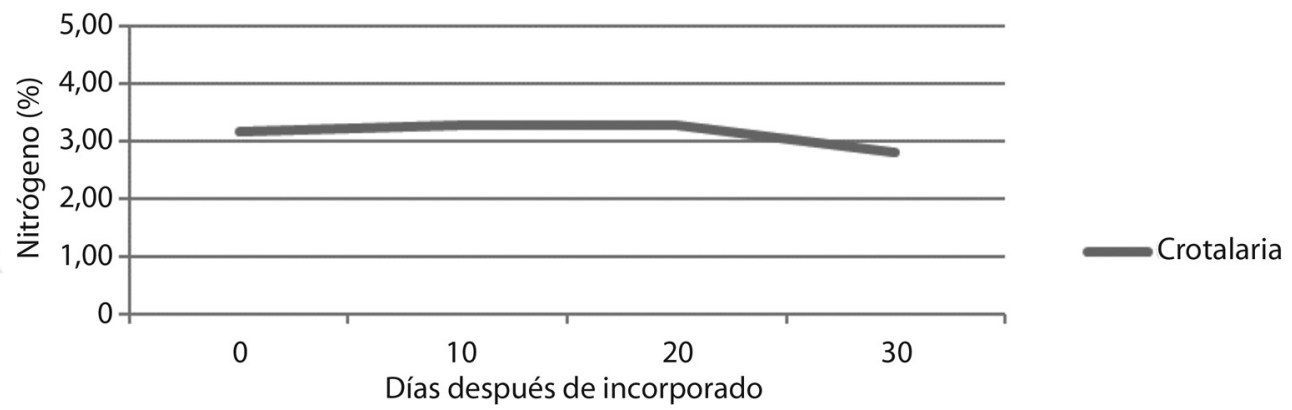

Figura 2. Mineralización de nitrógeno crotalaria del rastrojo incorporado en un suelo Andisol en Heredia. T2. Crotalaria.

19\% aproximadamente, siendo más alta que la especie Mucuna).

Al finalizar el periodo de mineralización, se pudo observar que existieron diferencias significativas entre los dos tratamientos de las especies Mucuna y Crotalaria utilizadas en los suelos Andisoles, especialmente con el rastrojo de Mucuna a los cero, veinte y treinta días de dicho periodo.
En cuanto a la mineralización que sufrió los tratamientos la especie Mucuna (Mucuna pruriens), (T3), durante los primeros 20 días de establecido el experimento figura 3 , la mineralización presentó un proceso lento igual al sufrido por el tratamiento en el que se utilizó Crotalaria, en este caso hubo muy poca variación entre el porcentaje inicial y los 20 días, pero que después del día 20 hasta el día 30 del

CuAdRo 1

\section{Diferencias en la mineralización y aporte de nitrógeno de Mucuna y Crotalaria en suelo Andisol (Heredia)}

\begin{tabular}{|c|c|c|c|c|c|c|c|c|}
\hline \multirow{2}{*}{ Tratamiento } & \multicolumn{8}{|c|}{ Tiempo (días) } \\
\hline & \multicolumn{2}{|c|}{0} & \multicolumn{2}{|c|}{10} & \multicolumn{2}{|c|}{20} & \multicolumn{2}{|c|}{30} \\
\hline Crotalaria Heredia & 3.17 & $A$ & 3.28 & $A$ & 3.28 & $A$ & 2.81 & A \\
\hline Mucuna Heredia & 3.89 & $B$ & 3.32 & $A$ & 4.19 & $B$ & 4.6 & B \\
\hline$p$-valor & $<0$. & & 0.2 & & $<0$. & & 0.2 & \\
\hline
\end{tabular}

Las letras diferentes $p<0,05$.

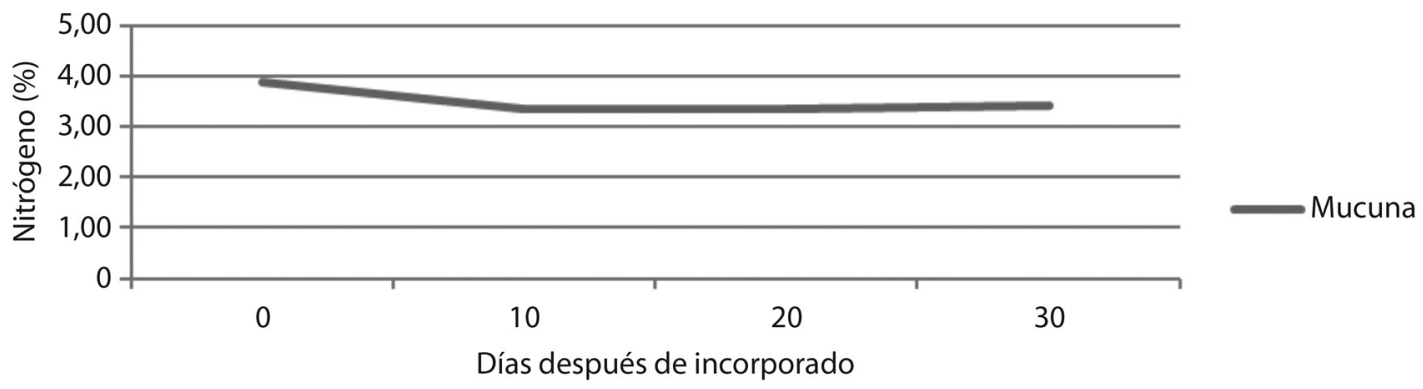

Figura 3. Mineralización y aporte de nitrógeno de Mucuna en un periodo de tiempo al incorporarlo en un suelo Ultisol. 


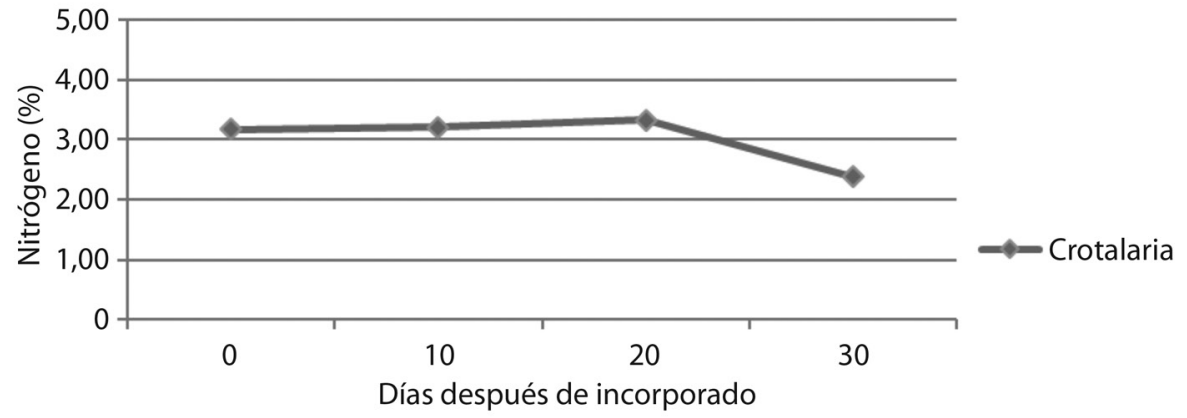

Figura 4. Mineralización y aporte de nitrógeno de Crotalaria en un periodo de tiempo al incorporarlo en un suelo Ultisol.

experimento, el contenido paso de un $4 \%$ a un $4,6 \%$, coincidiendo con (Rincón, 2016151 p), en donde expresa que aquellos rastrojos que presentan una relación C:N alta, son más lentos en su proceso de mineralización (Mucuna: C:N de un 14\%).

Finalizado el periodo de mineralización, se observó que existieron diferencias significativas entre los dos tratamientos de las especies Mucuna (T3) y Crotalaria (T2), utilizadas en los suelos de orden Andisol, específicamente con el rastrojo de Mucuna a los cero, veinte y treinta días de dicho periodo.

En la figura 3, la mineralización de los tratamientos (T3) de la especie Mucuna que se utilizó en suelos Ultisol, estos sufrieron un proceso de mineralización un poco lento, característico de los abonos verdes que poseen una relación $\mathrm{C} / \mathrm{N}$ alta, durante los primeros 10 días y a partir del día 10 y hasta el día 30 del experimento, el proceso de mineralización casi no sufrió ninguna variación de forma significativa en su aporte.
Mineralización de Crotoraría (Crotalaria juncea) en suelo Ultisol.

Como se observa en la figura 4, el proceso de mineralización de los tratamientos de la especie Crotalaria (T 4), fue lento durante los primeros 20 días de establecido el experimento, ya que sus valores se mantuvieron en un 3,3\% de $\mathrm{N}$.

A partir del día 20 del experimento (cuadro 2), el contenido de nitrógeno fluctuó de un $3,3 \%$ a un $2,4 \%$ aproximadamente es decir, hubo un aporte de un 0,9 $\%$ de nitrógeno a través de la mineralización de los tratamientos de la especie de Crotalaria, este cambio en la mineralización se pudo haber debido a que las condiciones climáticas y ambientales que imperaron en la zona de estudio coincidiendo con lo expresado por (García, 200619 p), que expresa que después de incorporar los rastrojos de leguminosas al suelo, estos se descomponen más rápidamente a causa de que los suelos tienen humedad suficiente para que ocurra el proceso de mineralización.

\section{CUADRO 2}

\section{Diferencias en la mineralización y aporte de nitrógeno de Mucuna y Crotalaria en suelo Ultisol (Pérez Zeledón)}

\begin{tabular}{|c|c|c|c|c|c|c|c|c|}
\hline \multirow{2}{*}{ Tratamiento } & \multicolumn{8}{|c|}{ Tiempo (días) \% } \\
\hline & \multicolumn{2}{|c|}{0} & \multicolumn{2}{|c|}{10} & \multicolumn{2}{|c|}{20} & \multicolumn{2}{|c|}{30} \\
\hline Crotalaria Pérez Zeledón & 3.17 & A & 3.21 & A & 3.32 & A & 2.38 & A \\
\hline Mucuna Pérez Zeledón & 3.89 & B & 3.35 & B & 3.36 & A & 3.43 & B \\
\hline$p$-valor & \multicolumn{2}{|c|}{$<0.0001$} & \multicolumn{2}{|c|}{0.0699} & \multicolumn{2}{|c|}{$<0.0001$} & $<0.0$ & \\
\hline
\end{tabular}


CUADRO 3

Aporte de nitrógeno en ambos suelos durante los 30 días de degradación de los rastrojos

\begin{tabular}{|c|c|c|c|c|c|}
\hline \multirow{2}{*}{ Variable } & & \multicolumn{2}{|c|}{ Heredia (Andisol) } & \multicolumn{2}{|c|}{ Pérez Zeledón (Ultisol) } \\
\hline & & Inicial & Final & Inicial & Final \\
\hline Relación C:N & & 11 & 1,20 & 8,00 & 1,17 \\
\hline $\mathrm{N}$ & $\%$ & 0,21 & 0,56 & 0,11 & 0,51 \\
\hline C & & 2,31 & 0,67 & 0,88 & 0,60 \\
\hline
\end{tabular}

En el cuadro 3, se coincide con (Cobo, 1998, 87 p), ya que la descomposición y liberación del nitrógeno generalmente es más lenta en residuos de abonos verdes que tienen una alta relación $\mathrm{C}$ : $\mathrm{N}$ y altos contenidos de lignina y polifenoles. El análisis final los tratamientos ( $\mathrm{T} 12,3$ y 4) de las especies de leguminosas en ambos suelos, lograron incrementar los porcentajes de nitrógeno, ya que en el suelo Andisol aumentó de $0,21 \%$ a 0,56 y en el suelo Ultisol se incrementó de 0,11 a $0,51 \%$.

Para los tratamientos (T 2 y 4) para la especie Crotalaria, aunque según los resultados esta especie produce un poco menos de nitrógeno también es una fuente importante de este suministro al suelo, estos tratamientos podrían aportar un promedio en Heredia de $188,27 \mathrm{Kg}$ N/ha y de $159,46 \mathrm{Kg} / \mathrm{N} / \mathrm{ha}$ en Pérez Zeledón aproximadamente.

\section{Conclusiones}

La mineralización de los tratamientos de Mucuna y Crotalaria es más efectiva durante los primeros 10 días de haberse depositado en los suelos Andisoles, pero que después este proceso suele seguir un comportamiento estático, en comparación con los depositados en suelos Ultisoles.

Los tratamientos de la leguminosa Mucuna se mineralizan más rápidamente que los rastrojos de los tratamientos de la especie Crotalaria en ambas zonas de estudio, debido a su menor porcentaje de celulosa y lignina.

Se concluye que a los 10 días de haber depositado los rastrojos en los suelos, es cuando se da la mayor mineralización de la especie Mucuna y aporte de nitrógeno en los suelos, aunque en menor porcentaje los tratamientos utilizados en la zona de Pérez Zeledón.

La cantidad de nitrógeno que podría ser fijado por los tratamientos de la especie Mucuna en
Heredia es de aproximadamente $367,7 \mathrm{Kg} \mathrm{n} \mathrm{N} / \mathrm{ha}$ y en la zona de Pérez Zeledón sería de 274,133 Kg $\mathrm{N} / \mathrm{ha}$ respectivamente.

\section{Recomendaciones}

Es necesario que se evalúe el efecto de las leguminosas como aportadoras de nitrógeno en suelos diferentes a los del presente estudio.

Para mejorar la productividad y calidad de los suelos, se recomienda el uso de abonos verdes, antes de implantar aquellos cultivos de nuestro interés, debido al gran aporte de nutrientes que proveen al suelo.

Se recomienda incorporar las leguminosas para aportar abono orgánico, cuando las condiciones de humedad del suelo sean mayor al $31 \%$.

Realizar nuevas investigaciones en zonas con similares características edafo-climáticas para comparar y validar la información obtenida en este estudio.

El uso de abonos verdes provenientes de leguminosas, es una práctica conservacionista que se debe de implementar en aquellos suelos donde se han utilizado gran cantidad de fertilizantes químicos.

Apoyar investigaciones donde se utilicen abonos verdes provenientes de leguminosas, con el objetivo de implementar producciones agrícolas más conservacionistas.

\section{Referencias Bibliográficas}

Cobo, J.(1998). Abonos verdes como fuentes de nitrógeno en un agroecosistema tropical de Ladera en Colombia. Catie. San José, Costa Rica. p.87.

Durango, W. (2014). Efecto de dosis crecientes de enmiendas orgánicas en un Andisol y un Ultisol, sobre la biomasa microbiana, respiración y actividad enzimática, en condiciones de invernadero. Tesis Msc. Ciencias Agrícolas y Recursos Naturales. UCR, Costa Rica. $137 \mathrm{p}$. 
García, M. (2006). Estudio comparativo de diferentes especies de abonos verdes y su influencia en el cultivo del maíz. Instituto Nacional de Ciencias Agrícolas (INCA), La Habana, Cuba. Volumen 23 (19).

InfoStat, (versión 2016). Manual del usuario. Grupo InfoStat,FCA, Universidad Nacional de Córdoba. Editorial Brujas, Argentina.

Moreno, J. (2009). Contenido nutrimental de tres especies de frijol producido mediante tres sistemas de labranza. Tesis Ing. Agrónomo. La Paz, Baja California Sur, México, 73 p.

Prager, M; Sanclemente, O; Sánchez de Prager; Miller, J; Ángel, D. (2012). Abonos verdes: Tecnología para el manejo agroecológico de los cultivos. Universidad Nacional de Colombia. Palmira Valle. Colombia, 53$62 \mathrm{p}$.
Rincón, E. (2016). Utilización de leguminosas forrajeras como abonos verdes para la producción de cultivos forrajeros en el trópico seco. Bogotá. Colombia, Universidad Nacional, Facultad de Medicina Veterinaria. Tesis, Doctor Ciencias ProducciónAnimal.151.p.

Ruiz, J. (2003). Evaluación de los abonos verdes en asociación con maíz de temporal en los valles centrales de Oaxaca, México. Terra Latinoamérica. 415. p. 